Case report

\title{
An uncommon therapeutic option for a challenging cause of pleural effusion
}

A 33-year-old nonsmoking Vietnamese man, without any past medical history was evaluated in another hospital in September 2018 for rapidly progressive shortness of breath. The chest radiograph showed a right pleural effusion. A thoracentesis was performed. On gross examination, the appearance of the pleural fluid was milky.

\section{Task 1}

What diagnosis do you suspect? Which test will confirm this diagnosis?
Cite as: Le Tuan L, Nguyen Ngoc C, Tran Viet H, et al. An uncommon therapeutic option for a challenging cause of pleural effusion. Breathe 2019; 15: e69-e76.

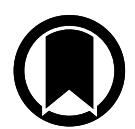

CrossMark 


\section{Answer 1}

The milky appearance of the pleural fluid suggests a chylothorax. Analysis of triglycerides in the pleural fluid will confirm this diagnosis.

Pleural fluid analysis showed a transudative fluid (pleural proteins $27 \mathrm{~g} \cdot \mathrm{L}^{-1}$, ratio of pleural proteins to blood proteins of 0.42 ). The triglyceride level was increased to $9.54 \mathrm{mmol} \cdot \mathrm{L}^{-1}$ (normal range: $<1.88 \mathrm{mmol} \cdot \mathrm{L}^{-1}$ ) and the cholesterol level was $2.29 \mathrm{mmol} \cdot \mathrm{L}^{-1}$ (normal range: $<5.2 \mathrm{mmol} \cdot \mathrm{L}^{-1}$ ).

Task 2

Which treatment will you initiate regardless of the cause?

\section{Answer 2}

The first step in management of a chylothorax is usually conservative: pleural drainage and dietary changes with an oral high-protein and low-fat diet (<10 g fat per day).

Pleural drainage with a large bore chest tube was performed with quick relief of the dyspnoea and an oral high-protein and low-fat diet was introduced.

The patient underwent clinical re-evaluation. In August 2018, he had had a motorbike accident and had fallen down on his right thorax, without any complications at the time (no rib fracture, no pneumothorax and no spinal injuries were found on the computed tomography (CT) scan of the thorax, abdomen, pelvis and whole spine, with contrast). He had no general status alteration, no respiratory or digestive symptoms, and no recent invasive medical or surgical procedure. On physical examination, he had no lymphadenopathy, no lymphedema, no goiter, no skin or joint abnormalities, no malformations, and no sign of superior vena cava syndrome.

Task 3

Which cause of chylothorax is most probable in this patient? Which following complementary examination will you perform? 


\section{Answer 3}

The most probable cause of chylothorax in this patient is a blunt thoracic trauma. A CT scan of the thorax and abdomen should be performed to exclude other diagnoses (e.g. mediastinal lymphadenopathy or mass, substernal goiter, pulmonary cysts).

A CT scan of the thorax and abdomen showed a right pleural effusion with the chest tube in the correct position, but no rib fracture or chest wall lesion. Lung parenchyma, mediastinum and abdomen were normal.

However, despite 2 months of optimal medical treatment, the fluid output remained 600$800 \mathrm{~mL} \cdot$ day $^{-1}$. The patient was then referred to our hospital for further management in November 2018.

On arrival, his physical examination was normal. His blood tests were remarkable for mild hypoalbuminaemia, mild hyponatraemia and positive $\mathrm{HbS}$ antigen (table 1 ).

The chest radiograph and the CT scan of the thorax (figure 1) showed a remaining mild right pleural effusion, without any other lesion. The chest tube was in a correct position.

\section{Task 4}

What treatment options should be discussed?
Table 1 Blood tests

\begin{tabular}{|c|c|c|}
\hline Test & Result in patient & Normal values \\
\hline White blood cells $\times 10^{9} \mathrm{~L}^{-1}$ & 5.47 & $4-10$ \\
\hline Haemoglobin $\mathrm{g} \cdot \mathrm{dL}^{-1}$ & 13.3 & $12.5-17.5$ \\
\hline Platelets $\times 10^{9} \mathrm{~L}^{-1}$ & 233 & $150-450$ \\
\hline Prothrombin \% & 82 & $70-140$ \\
\hline Proteins $g \cdot \mathrm{L}^{-1}$ & 63.4 & $66-87$ \\
\hline Albumin $\mathrm{g} \cdot \mathrm{L}^{-1}$ & 27 & $35-52$ \\
\hline Sodium $\mathbf{m m o l} \cdot \mathrm{L}^{-1}$ & 131 & $136-145$ \\
\hline Potassium $\mathrm{mmol} \cdot \mathrm{L}^{-1}$ & 4.1 & $3.5-4.5$ \\
\hline Chlorine $\mathrm{mmol} \cdot \mathrm{L}^{-1}$ & 95 & $98-107$ \\
\hline Creatinine $\mu \mathrm{mol} \cdot \mathrm{L}^{-1}$ & 81 & $62-106$ \\
\hline ASAT U. $\mathbf{L}^{-1}$ & 27 & $<40$ \\
\hline ALAT $\mathbf{U} \cdot \mathbf{L}^{-1}$ & 36 & $<41$ \\
\hline HIV (Elisa test) & Negative & \\
\hline HCV antibodies & Negative & \\
\hline HBV antigen HbS & Positive (378 COI) & $<0.9$ \\
\hline Filariasis antibodies & Negative & \\
\hline
\end{tabular}

ASAT: aspartate amino transferase; ALAT: alanine amino transferase; HCV: hepatitis C; HBV: hepatitis B; COI: cut off index.
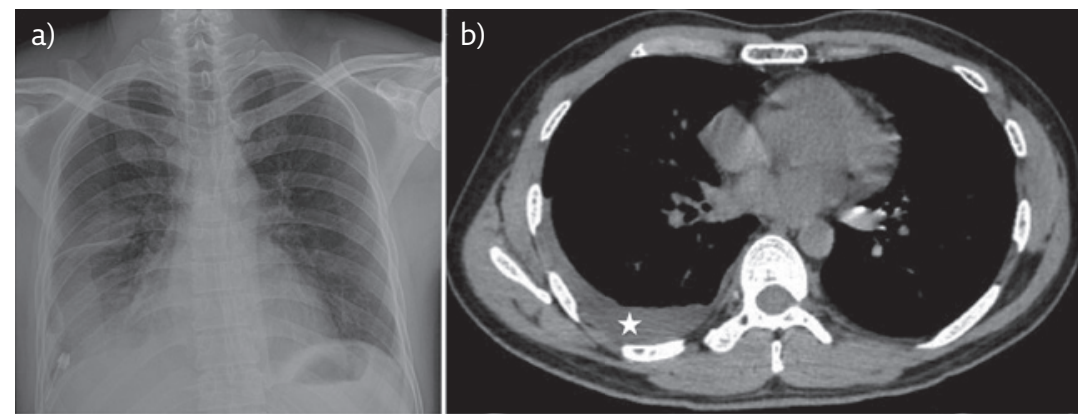

Figure 1 a) Chest radiograph and b) CT scan of the thorax in November 2018, showing a remaining mild right pleural effusion with an $800 \mathrm{~mL}$ daily output, despite 2 months of adequate chest tube drainage and a high-protein and low-fat diet. 

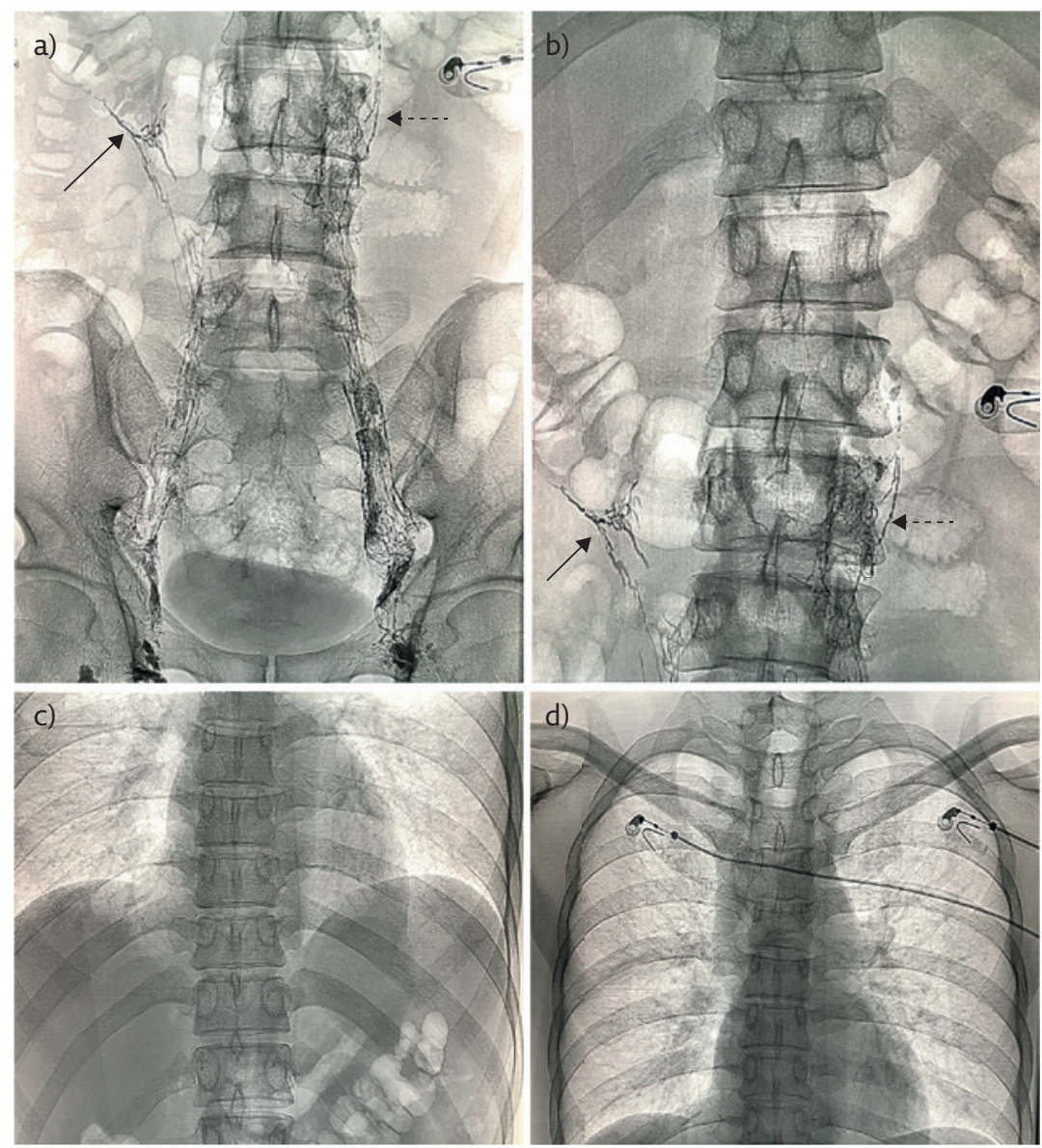

Figure 2 Intranodal lymphangiography. a, b) Cisterna chyli are not seen. Tiny lymphatic vessels are going up (dashed arrows), most visible around the right kidney (full arrow). c, d) no lymphatic vessels are seen at the thoracic level.

\section{Answer 4}

Octreotide treatment and total parenteral nutrition can be discussed as a first approach. For patients who fail conservative therapy, a surgical or a radiological intervention is warranted. The surgical options are a thoracic duct ligature or a pleurodesis during a thoracoscopy. The alternative is a thoracic duct embolisation by interventional radiology.

In our patient, total parenteral nutrition over 1 week did not change the volume of leak.

An intranodal lymphangiography (figure 2) was performed to localise the site of chyle leak and examine lymphatic anatomy. This procedure showed no visibility of cisterna chyli, but tiny lymphatic vessels going up separately predominant around the right kidney. After $1 \mathrm{~h}$, there was no contrast flow at the thoracic level.

$12 \mathrm{~h}$ after lymphangiography, a CT scan of the thorax showed no contrast material in the lymphatic vessels at the thoracic level. Another non-contrast $\mathrm{CT}$ scan of the thorax was carried out $24 \mathrm{~h}$ after lymphangiography (figure 3): it showed many lymphatic vessels around the right infero-posterior parietal pleura and around the inferior vena cava, but no visibility of the thoracic duct.

\section{Task 5}

Based on the lymphangiography results, which therapeutic options can be discussed?
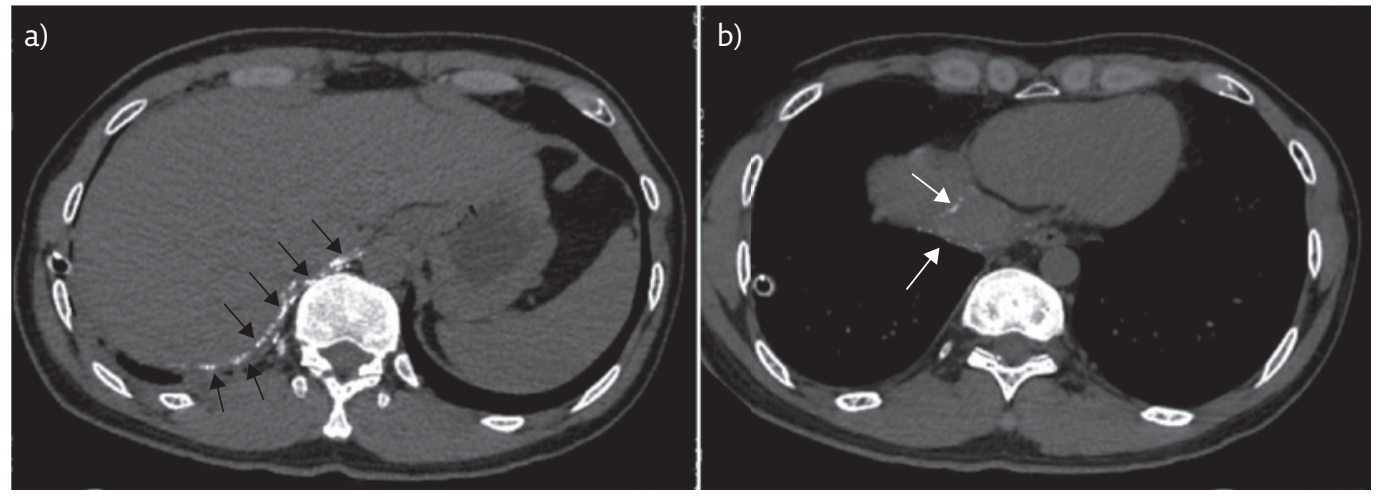

Figure 3 CT scan of the thorax $24 \mathrm{~h}$ after intranodal lymphangiography. Lymphatic vessels a) around the right inferoposterior parietal pleura (black arrows) and b) around the inferior vena cava (white arrows). 


\section{Answer 5}

Because of the variant anatomy, the therapeutic options are a pleurodesis, a tunnelled drain, a peritoneal shunt or a percutaneous puncture of the visible right thoracic vessels.

After multidisciplinary discussion involving pulmonologists, thoracic surgeons and interventional radiologists, an interventional radiology procedure was decided upon. A surgical ligature of the thoracic duct was not indicated because of the variant anatomy (no visibility of the thoracic duct). Pleurodesis was discussed; however, a less invasive and less expensive option was preferred. Tunnelled drain and peritoneal shunt were ruled out because in this young and active patient a therapeutic rather a palliative option was more appropriate.

The interventional radiology procedure involved a percutaneous puncture of the visible right thoracic lymphatic vessels under CT guidance and local anaesthesia, using a 22G needle, followed by injection of absolute ethanol to obtain sclerotherapy (figure 4).

The day after, the output decreased significantly to $<50 \mathrm{~mL} \cdot$ day $^{-1}$. The chest tube was removed after 3 days, without any recurrence of pleural effusion during the week. During this time, the patient continued to receive total parenteral nutrition. The patient had no side-effects from the procedure and was discharged home with advice to continue a low-fat and high-protein diet.

2 months later, in January 2019, the patient was re-evaluated. He was asymptomatic and had started to work again without any problems. His physical examination and nutritional status were normal. The CT scan of the thorax (figure 5) showed no recurrence of pleural effusion. He was advised to stop the high-protein and low-fat diet.

\section{Discussion}

Chylothorax is a rare cause of pleural effusion due to leaking of chyle from the thoracic duct or from an
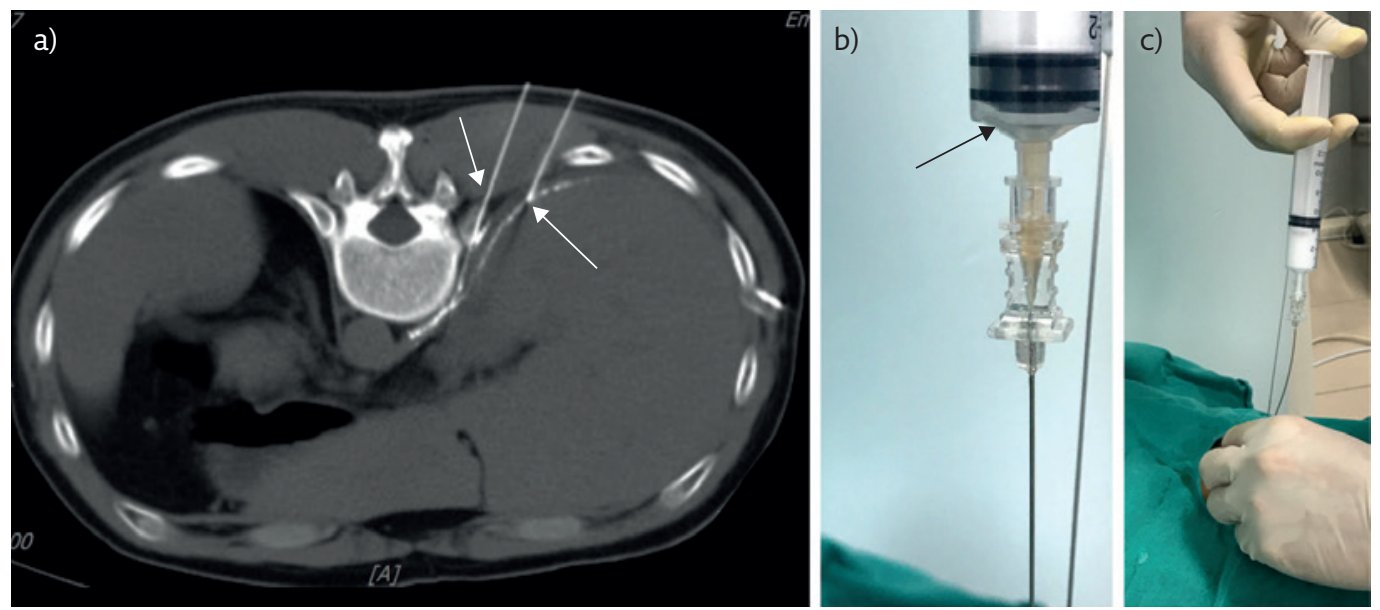

Figure 4 Percutaneous embolisation of lymphatic vessels. a) The 22G needle (Chiba, Cook Company, USA) is inserted percutaneously under CT guidance (arrows) into the lymphatic vessel, which was already opacified by lymphangiography. b) Aspiration of lymph in the syringe (arrow) confirms that the tip of needle is within the lymphatic vessel. c) Absolute ethanol is injected slowly through the needle into the lymphatic vessel until the patient feels pain, indicating that the ethanol has reached the pleural space. For this procedure, a total of $8 \mathrm{~mL}$ absolute ethanol was injected.
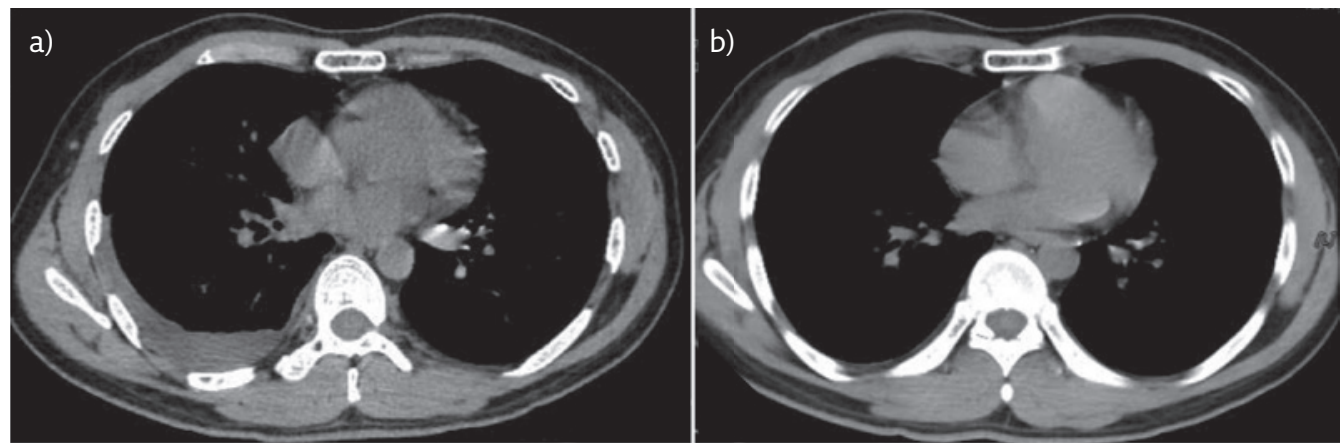

Figure 5 CT scan of the thorax without contrast. a) CT scan of the thorax in November 2018, before sclerotherapy showing mild right pleural effusion. b) CT scan of the thorax in January 2019, 2 months after sclerotherapy showing no recurrence of right pleural effusion. 
afferent thoracic lymphatic vessel into the pleural space.

Chyle is formed in the intestines and contains proteins, lipids, electrolytes and lymphocytes. It is carried through the thoracic duct to the bloodstream. The thoracic duct starts with the cisterna chyli at the second lumbar vertebra, passes through the mediastinum where it receives lymph from lymphatic vessels draining the pulmonary parenchyma and parietal pleura, and ends at the junction of the left subclavian and jugular veins (figure 6) [1, 2]. The total lymphatic flow through the thoracic duct is around 1500-2400 mL day ${ }^{-1}$, but increases after rich fatty meals (especially those containing long-chain triglycerides). Any disruption of this flow can cause chylothorax: a lesion of the thoracic duct or a lesion of an afferent thoracic lymphatic vessel, and it can occur after a major thoracic surgery such as lobectomy with mediastinal lymph node dissection. Chronic chyle leaks result in metabolic disorders, malnutrition, thromboembolic disease, impaired immunity and difficulty to cicatrise.

The clinical manifestations of chylothorax are poor, usually a progressive dyspnoea, tiredness and signs of malnourishment. The chest radiograph shows a pleural effusion.

The diagnosis of chylothorax is confirmed by a thoracentesis which shows [2-5]: a milky fluid on gross examination with increased levels of triglycerides $\left(>110 \mathrm{mg} \cdot \mathrm{dL}^{-1}\right.$ or $>1.24 \mathrm{mmol} \cdot \mathrm{L}^{-1}$ ), a ratio of pleural fluid to serum triglycerides of $>1$, and a low cholesterol level $\left(<200 \mathrm{mg} \cdot \mathrm{dL}^{-1}\right.$ or $<5.18 \mathrm{mmol} \cdot \mathrm{L}^{-1}$ ) with a ratio of pleural fluid to pleural cholesterol of $<1$. If there is a doubt, the presence of chylomicrons, identified by lipoprotein electrophoresis, is the gold standard.

The pleural fluid can be exudative or transudative, with a predominance of lymphocytes and is sterile.
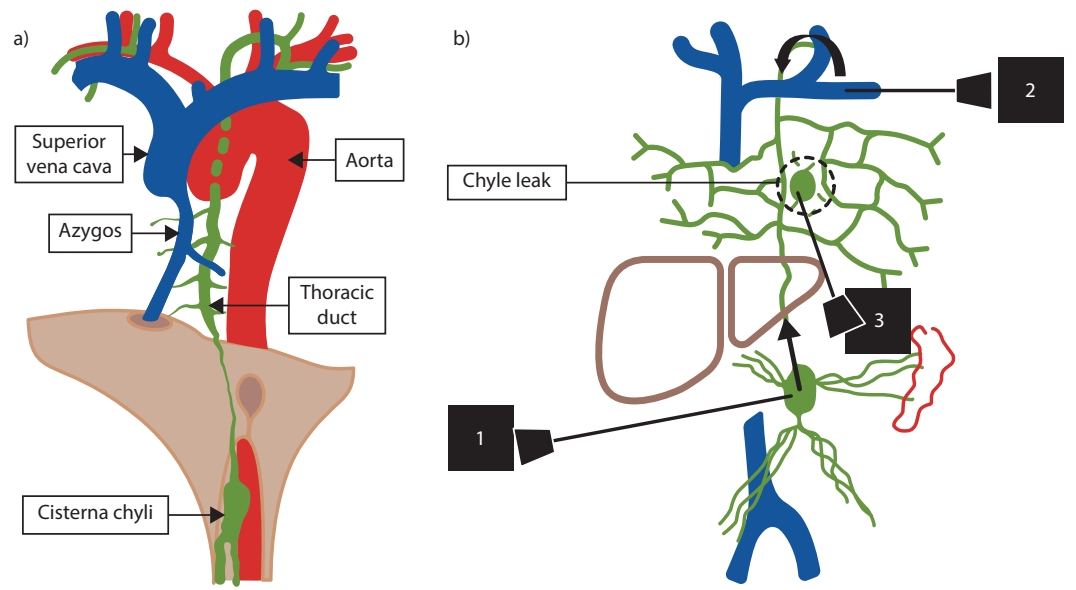

Figure 6 Thoracic duct anatomy and different routes of access for sclerotherapy. a) Simplified thoracic duct anatomy. b) Different routes of access to perform sclerotherapy for chylothorax: 1) transabdominal thoracic duct embolisation by catheterisation of the thoracic duct via the cisterna chyli; 2) retrograde transvenous embolisation of the thoracic duct through the lymphovenous junction; and 3) direct embolisation of the abnormal lymphatic vessel.
Differential diagnosis of chylothorax includes pseudochylothorax (ratio of pleural fluid to pleural cholesterol level of $>1$ ), due to a chronic exudative pleural effusion such as chronic tuberculosis pleurisy and empyema.

The causes of chylothorax (table 2) are traumatic or nontraumatic interruption of the flow of chyle through the thoracic duct $[2,4,5]$. The iatrogenic traumatic causes occur after thoracic surgery, most often surgery of the oesophagus, or after radiotherapy. The other traumatic causes are due to penetrant or blunt thoracic trauma. The nontraumatic causes include cancers (lymphoma, cancer of the thoracic cavity), diseases obstructing the thoracic duct or leading to aberrant lymphatic flow (e.g. sarcoidosis), infections such as tuberculosis, lymphadenopathy, or congenital diseases.

The first step of treatment for a chylothorax is medical [2, 4-6]: chest tube drainage and a lowfat diet $\left(<10 \mathrm{~g} \cdot \mathrm{day}^{-1}\right)$, rich in proteins, without long-chain triglycerides but rich in medium-chain triglycerides [7, 8]. This diet may be replaced by parenteral nutrition alone. If the cause is nontraumatic, treatment of the underlying cause is required.

If there is high output of chyle $\left(>1000 \mathrm{~mL} \cdot\right.$ day $\left.^{-1}\right)$, a persistence of output $>500 \mathrm{~mL} \cdot$ day $^{-1}$ over 2 weeks or an underlying neoplastic aetiology, invasive treatment should be considered: either surgical treatment or interventional radiology [2, 4-6]. In this case report, there was a 2-month delay before invasive treatment, exposing the patient to malnutrition (reflected by his low albumin level on arrival) and social problems because he was not able to work while hospitalised. Understanding the usual evolution of chylothorax and the criteria for medical treatment failure is therefore indispensable to decide the best therapeutic option, with respect to body, mental and social health.

Surgical treatment of chylothorax is often successful [9, 10], and includes thoracic duct ligature, pleurodesis, and less often, tunnelled drains or pleural shunt procedures. However, a surgical procedure may be at high risk of complications in these patients with a status of malnutrition and mild immunosuppression, and can be technically difficult because of variant anatomy, such as absence of the thoracic duct, or high output leading to failure of pleurodesis. In this case report, thoracic ligation of the thoracic duct was not feasible because the thoracic duct was not seen during lymphangiography. Pleurodesis, tunnelled drain and peritoneal shunt were ruled out as explained in the case report.

Thoracic duct embolisation is a minimally invasive radiological procedure, performed percutaneously, under local anaesthesia, which allows direct embolisation of the thoracic duct with a sclerotic agent. It is an efficient alternative to surgical treatment of chylothorax, with a reported success rate of $70-90 \%$ [11-15]. There are very 
few acute complications, which are usually minor (perihepatic haematoma, guidewire fragments sheared off in the retroperitoneum, non-target embolisation into the lungs or adjacent vessels of the thoracic duct) $[4,15,16]$. Reported delayed complications are chronic diarrhoea and lower extremity swelling [17].

Prior to thoracic duct embolisation, because lymphatic leakage can occur anywhere along the pathway of lymph, a lymphangiography is needed to localise the leak and detect variant anatomy. Nowadays, the complicated traditional pedal lymphangiography has been replaced with an easier method: intranodal lymphangiography $[4,16$, $18,19]$. An inguinal lymph node is directly accessed under ultrasound guidance, and a lipiodol injection is performed through a needle. Radiographic images are obtained thereafter to follow the course of lipiodol through the chyle pathway. A follow-up non-contrast CT scan of the thorax is obtained to better assess the site of leakage and identify an appropriate puncture site for sclerotherapy.

There are two classical routes of access to perform embolisation of the thoracic duct (figure 6): through the cisterna chyli or by retrograde cannulation of the thoracic duct through the left subclavian vein. However, in the case of our patient, neither the thoracic duct nor cisterna chyli were visible. The leak appeared to come from the lymphatic vessels draining the right parietal pleura, because of accumulation of lipiodol at that site. So, a direct embolisation of these structures was successfully performed, without any complication, allowing the patient to return quickly to a normal life. Hur et al. [20] described this rare technique of direct upstream lymphatic vessel embolisation in 2016.

This case report highlights the efficiency and safety of minimally invasive interventional radiology as an alternative to invasive surgery; and the importance of intranodal lymphangiography prior to these procedures, in order to localise the site of the leak and to detect variant anatomy, which will enlighten the final therapeutic decision. Moreover, it describes a recent and rare direct embolisation technique of the lymphatic vessels.

Table 2 Main causes of chylothorax

\section{Traumatic}

latrogenic

Non-iatrogenic

\section{Nontraumatic}

Malignant

Non-malignant obstruction of the thoracic duct or aberrant lymphatic flow

位

Congenital

Infectious diseases

\begin{tabular}{ll} 
Histoplasmosis & Mediastinitis \\
& Filariasis \\
Idiopathic & \\
\hline
\end{tabular}

Thoracic and cervical surgery

Oesophagus surgery

Retroperitoneal surgery

Cervical vascular catherisation

Thoracic irradiation

Penetrant and non-penetrant trauma

Increased thoracic pressure (peripartum, heavy cough or vomiting)

Lymphoma, chronic lymphocytic leukaemia, multiple myeloma

Lung cancer, oesophagus cancer, mediastinal metastasis of other cancers

Kaposi sarcoma

Sarcoidosis

Amyloidosis

Superior vena cava thrombosis

Lymphangioleiomyomatosis

Castleman disease

Goiter

Constrictive pericarditis

Yellow-nail syndrome

Chylous ascites

Turner syndrome

Noonan syndrome

Tuberculosis

tinitis

Filariasis

\section{Affiliations \\ Linh Le Tuan ${ }^{1}$, Cuong Nguyen Ngoc ${ }^{1}$, Hung Tran Viet ${ }^{1}$, Hoan Le ${ }^{2}$, François Pons ${ }^{3}$, Delphine Natali ${ }^{4}$ \\ ${ }^{1}$ Radiology Dept, Hanoi Medical University Hospital, Hanoi, Vietnam. ${ }^{2}$ Respiratory Medicine Dept, Hanoi Medical University Hospital, Hanoi, Vietnam. ${ }^{3}$ Visceral and Thoracic Surgery Dept, Hanoi French Hospital, Hanoi, Vietnam. ${ }^{4}$ Respiratory Medicine Dept, Hanoi French Hospital, Hanoi, Vietnam.}

\section{Conflict of interest}

None declared. 


\section{References}

1. Achour K, Riquet M. Anatomie chirurgicale des lymphatiques du thorax. Chir Thorac Cardiovasc 2011; 15: 37-44.

2. Nair SK, Petko M, Hayward MP. Aetiology and management of chylothorax in adults. EurJ Cardiothorac Surgery 2007; 32: 362-369.

3. Staats BA, Ellefson RD, Budahn LL, et al. The lipoprotein profile of chylous and nonchylous pleural effusions. Mayo Clin Proc 1980; 55: 700-704.

4. Murrey DA, Kapoor BS, Cain TR, et al. ACR appropriateness criteria chylothorax treatment planning. J Am Coll Radio/ 2017 14: S118-S126.

5. McGrath EE, Blades Z, Anderson PB. Chylothorax: Aetiology, diagnosis and therapeutic options. Respir Med 2010; 104: 1-8.

6. Bender B, Murthy V, Chamberlain RS. The changing management of chylothorax in the modern era. Eur $J$ Cardiothorac Surgery 2016; 49: 18-24.

7. Sriram K, Meguid RA, Meguid MM. Nutrition support in adults with chyle leaks. Nutrition 2016; 32: 281-286.

8. Takuwa T, Yoshida J, Ono S, et al. Low-fat diet management strategy for chylothorax after pulmonary resection and lymph node dissection for primary lung cancer. J Thorac Cardiovasc Surg 2013; 146: 571-574.

9. Paul S, Altorki NK, Port JL, et al. Surgical management of chylothorax. Thorac Cardiovasc Surg 2009; 57: 226-228.

10. Ferguson MK. Thoracoscopy for empyema, bronchopleural fistula, and chylothorax. Ann Thorac Surg 1993; 56: 644-645.

11. Cope C, Salem R, Kaiser LR. Management of chylothorax by percutaneous catheterization and embolization of the thoracic duct: prospective trial. J Vasc Interv Radiol 1999; 10 1248-1254.
12. Cope C, Kaiser LR. Management of unremitting pneumothorax by percutaneous embolization and blockage of retroperitoneal lymphatic vessels in 42 patients. J Vasc Inter Radiol 2002; 13: 1139-1148

13. Binkert CA, Yucel EK, Davidson BD, et al. Percutaneous treatment of high-output chylothorax with embolization or needle disruption technique. J Vasc Interv Radiol 2005; 16: 1257-1262.

14. Pamarthi V, Stecker MS, Schenker MP, et al. Thoracic duct embolization and disruption for treatment of chylous effusions: experience with 105 patients. J Vasc Interv Radiol 2014; 25: 1398-1404

15. Kim PH, Tsauo J, Shin JH. Lymphatic interventions for chylothorax: a systemic review and meta-analysis. J Vasc Interv Radiol 2018; 29: 194-202.

16. Stecker MS, Fan CM. Lymphangiography for thoracic duct interventions. Tech Vasc Interventional Rad; 19: 277-285.

17. Laslett D, Trerotola SO, Itkin M. Delayed complications following technically successful thoracic duct embolization. J Vasc Interv Radiol 2012; 23: 76-79.

18. Nadolski GJ, Itkin M. Feasibility of ultrasound-guided intranodal lymphangiogram for thoracic duct embolization. J Vasc Interv Radiol 2012; 23: 613-616.

19. Lee EW, Shin JH, Ko HK, et al. Lymphangiography to treat postoperative lymphatic leakage: a technical review. Korean J Radiol 2014; 15: 724-732.

20. Hur S, Shin JH, Lee IJ, et al. Early experience in the management of postoperative lymphatic leakage using lipiodol lymphangiography and adjunctive glue embolization. J Vasc Interv Radiol 2016; 27: 1177-1186. 